\title{
Child psychiatric epidemiology: stars and hypes
}

\author{
Frank C. Verhulst $\cdot$ Henning Tiemeier
}

Published online: 20 May 2015

(C) Springer-Verlag Berlin Heidelberg 2015

Epidemiological studies have been essential for the development of child psychiatry over the last half-century, especially in describing the frequency and course of child psychiatric problems, and in demonstrating strong crossculturally consistent associations between social disadvantage or child neglect with child psychopathology. Traditionally, epidemiological studies also attempt to tackle questions of causality of child mental health problems. For obvious ethical and practical reasons, the experimental design to answer causal questions is often not feasible, and observational approaches, more than ever, have been crucial for a better understanding of the role of risk factors in the development of psychopathology. Perhaps the most outstanding contribution of recent epidemiological science has been to show the many reasons why observed associations do not reflect environmentally mediated causal effects. For example, the association between prenatal smoking and later child problem behaviours can largely be explained by inherited factors transmitted from mother to child [1].

So far so good, and surely this special issue presents a number of contributions, including the ones on the BELLA cohort, to this good old epidemiological tradition [2]. But what is new, what promising scientific developments do we need to follow closely, and who are the stars that will shape the future? Equally important is the question whether we can identify hypes in child psychiatric epidemiology that make researchers blindly follow the flavours of the month and put their money into activities that may be spent better in more promising areas of research?

F. C. Verhulst $(\square) \cdot$ H. Tiemeier Department of Child and Adolescent Psychiatry, Erasmus Medical Center-Sophia Children's Hospital, P.O. Box 2060, 3000 CB Rotterdam, The Netherlands

e-mail: f.verhulst@erasmusmc.nl
Usually, epidemiologists stay away from trying to forecast the future at the individual level. One of today's most influential epidemiologists, George Davy Smith, emphasizes the neglected role of chance events that contributes a stochastic element to all occurrences and hampers meaningful prediction of any specific negative or positive outcome [3]. In this editorial, we take the liberty, admittedly with some pleasure, to violate his advice and attempt to predict the future of child psychiatric epidemiology. We hope the reader will appreciate that we briefly look ahead and try to identify those epidemiological activities and study opportunities that, to our opinion, are exciting and deemed successful, as well as those that may well lead to dead-ends.

Studies need to look for innovative opportunities to test hypotheses rather than put their ambition in unrealistic large size only. A recent dramatic example of how an epidemiological study can fail is the dismantling of the National Children's Study (NCS) in December 2014. This study aimed to follow the health of 100,000 U.S. children from before birth to age 21 and investigate a range of hypotheses developed by hundreds of scientists on the influences of countless factors, from chemical to psychosocial exposures, on child development and health outcomes. This study cost more than $\$ 1.2$ billion, but because of its scientific and managerial flaws it was discontinued after only some pilot inclusions. This contrasts with studies of less ambitious size that are equally or more innovative and suited to test specific hypotheses. Examples are studies carried out in third world countries, where exposure to poverty is much greater than in Western countries, or studies in Scandinavian countries, where the use of registers with data on millions of individuals enables researchers to perform prospective etiological studies of disorders with relatively low frequencies such as autism. 
We identified an arbitrary number of six themes and their accompanying samples that, to our opinion, may particularly shape the near future of epidemiological research in the field of child and adolescent psychopathology. These themes are: (1) population neuroimaging studies; (2) largescale cross-disorder cohort studies; (3) preconception cohort studies; (4) resilience studies, (5) large genetic studies, and (6) modern registries.

Since the brain is involved in behavioural, emotional and cognitive functioning, neuroimaging studies may elucidate the mediating role of the brain in the association between risk factors and mental health problems. However, to date the best neuroimaging studies are cross-sectional or used small and selected samples, which make them vulnerable to a host of methodological problems including reverse causality, selection bias, and increased type I error due to multiple testing. Large-scale longitudinal population studies with repeated brain images and measurements of risk factors as well as behavioural and cognitive phenotypes are needed to determine associations between risk factors and changes in brain structure and function as well as the mediating role of the brain in the association between risk and behaviour or cognition. Consequently, the US National Institutes of Health recently announced funding opportunities for the Adolescent Brain Cognitive Development (ABCD) study consortium. With a funding of $\$ 25,000,000$ for 2015 alone and equal funding for each of the coming five years, this future multisite project aims to study the impact of substance use on the structure and function of the developing brain across adolescence. This study will undoubtedly be the star study investigating the effects of substance use on adolescent brain, behavioural and cognitive development.

Large-scale cross-disorder studies aim to determine how economic, social and biological factors from the prenatal period jointly explain human behaviour in key important areas, including child development and mental health problems in general. The UK has a tradition of very successful large-scale birth cohort studies, and the Life Study is an ambitious next-generation cohort following 80,000 pregnant mothers in the UK and their children born between 2014 and 2018. The size of the sample will constrain the detail of the behavioural phenotypes to be assessed, but in-depth measures are planned and the strength of the British studies lies in the power to detect the effects of risks on child developmental problems across traditional psychiatric diagnostic categories.

Most cohort studies start to recruit mothers in pregnancy at about 12 weeks of gestation. To evaluate exposures of mothers and fathers in the pre-conceptual period and those around the time of conception, these cohort studies start too late. Participants should be included in the preconception period to study the association of risk exposures with gamete and embryo quality and ultimately the development of children after birth. It is a challenge to obtain a sizeable sample before conception, thus few if any large preconception cohorts exist. GUSTO, a birth cohort launched in Singapore in 2009, failed to include many women before pregnancy. Similarly, the failed National Children's Study intended to include about $25 \%$ of its participants before conception. Hopefully the plan to include women before pregnancy was not key to this study's failure. Preconception studies are needed to study the effects of exposures such as exposure to persistent environmental chemicals.

Most existing cohort studies are carried out in highincome countries with relatively low levels of exposures to severe social adversities. Birth cohort studies from low- and middle-income countries, including Brazil, Guatemala, India, Philippines, and South Africa [4] include study populations that experience high levels of violence, and physical and sexual abuse. For example, it is estimated that $40 \%$ of South African women will be raped in their lifetime, and Guatemala is listed amongst countries with the highest homicide rates. Growing-up in (many parts of) these countries is a challenge and individuals who end up relatively unscathed must have been able to build up resilience against the risks associated with such dangerous and distressing circumstances. The Pregnancy Outcomes, Maternal and Infant Study (PrOMIS) Cohort from Lima, Peru [5] and the 1982 Pelotas Brazil Birth Cohort Study [6] are, sadly enough, examples of studies of populations with extremely high exposures to violence, abuse and maternal mental health problems. These populations offer ample opportunity to study risks and resilience in adverse environments such as those characterized by intimate partner emotional and physical violence.

What about genetics? Mental illness in a parent is a very strong, but unspecific, risk factor for mental illness in the offspring. However, attempts to unravel the molecular genetic basis of psychiatric disorders over the last decade, such as genome wide association studies (GWAS), have not yet been very successful, or as Uher and Rutter sceptically state in 2012: "....molecular genetic studies of psychiatric disorders have done a lot to find very little" [7]. The overview on genetics in child and adolescent psychiatry by Hohmann et al. [8] in this special issue takes a more positive standpoint and concludes that molecular genetic research has provided an increasing number of candidate genes possibly implicated in the pathophysiology of child and adolescent psychopathology. Different techniques within larger samples and with modified study designs are needed to overcome the missing heritability gaps. The authors propose data analytic techniques such as genetic network analyses on GWAS data, as well as the use of phenotypes reflecting unitary, cross-diagnostic functions, that are better suited to test the pleiotropic effects of genes 
than classical psychiatric diagnoses. Such phenotypes are dimensions such as emotional liability or neural circuitries underlying such functions. Here is where middle-sized cohort studies such as ALSPAC and GenerationR [9] come in, because they do not only have the genetic data; they also have the advantage of detailed phenotypic data. Arguably, GenerationR stands out because of its observational as well as neuroimaging data. Data pooling and replication across these types of cohorts may inform us about the genetic architecture of common forms of child and adolescent psychopathology.

The Scandinavian countries are well known for their nationwide population-based register data. The statistical power of these registries is evident and has the advantage to detect risks associated with uncommon conditions. An example is the Danish Medical Birth Registry, which maintains comprehensive medical records of every child. Linkage with data from other registries provides a wealth of epidemiological data, especially for conditions that infrequently occur in the general population, such as autism, but also for investigating trends in prescriptions of psychotropic medication for children and adolescents as exemplified by Steinhausen in this special issue [10]. Another very innovative example is The Finnish Prenatal Study of Autism or Schizophrenia, which are nested case-control studies based on maternal prenatal serum and linkages of nationwide registries on all children born in Finland between 1987 and 2000. The children were followed for the diagnosis of ASD and the availability of a wealth of medical information present for the more than 4000 (!) children who did and for those who did not receive this diagnosis, opens up ample opportunities to look for associations that may lead us to a better understanding of such disorders [11].

If we were to put money into epidemiological studies that, to our opinion, hold the most promise to advance the field of child and adolescent psychiatry, these are the themes and designs we would choose. We think that these efforts can move the field forward through better understanding of etiological mechanisms that are crucial for improving mental health in children and adolescents. Epidemiological knowledge has inspired public health interventions aimed at preventing mental health problems or stimulating healthy development in children and adolescents [12]. However, efficient household managing means that we can spend the money only once. Therefore, it is only fair if we also pick those themes that should receive less money, despite the fact that they are currently popular among funding bodies and scientists. Again, we hope the reader does not mind the liberty we take in this editorial by sharing our opinions freely. The five themes we, arbitrarily, identified as hypes in child psychiatric epidemiology are:

(1) candidate gene by environment interaction research;

(2) candidate gene epigenetics; (3) microbiome studies;
(4) psycho-immunology, and (5) studies testing the Barker hypothesis.

Since the ground-breaking work of Caspi et al. on candidate $\mathrm{G} \times \mathrm{E}(\mathrm{cG} \times \mathrm{E})$ interaction, hundreds of $\mathrm{cG} \times \mathrm{E}$ studies have been published. It is commonplace for teachers to use the $\mathrm{cG} \times \mathrm{E}$ studies by Caspi et al. as illustrative material, despite the fact that several researchers provided convincing argumentation that most or all positive $\mathrm{cG} \times \mathrm{E}$ reports are likely to be type I errors [13]. Modern genetic research shows that individual variants exert only small phenotypic effects. It also shows that risk loci from GWAS are usually found in unexpected, so-called intergenic, spaces of genes. This greatly limits the ability to correctly specify candidate hypotheses in addition to the problem that power is a major limitation in most $\mathrm{cG} \times \mathrm{E}$ studies given the small magnitude of genetic main effects. We think that the traditional $\mathrm{cG} \times \mathrm{E}$ concept is out-dated given the emerging evidence from genetic studies showing the complexity of the biological processes that are involved. The likelihood that $\mathrm{cG} \times \mathrm{E}$ studies using candidate genes that are the "usual suspects" for genetic variables will give us insights into complex genetic disorders such as psychiatric disorders is so small that we think we should finally abandon these types of studies if not based on GWAS data. Importantly, $\mathrm{cG} \times \mathrm{E}$ studies have taught us that solely presenting discovery findings is not sufficient and that molecular genetic $G \times E$ studies need to be accompanied by immediate replication efforts.

Epigenetic epidemiological studies primarily focus on exploring if and where the epigenome (i.e. the overall epigenetic state of a cell) is influenced by specific environmental exposures. Such knowledge may help us better understand the biological mechanisms involved in the effects of environmental influences such as prenatal nutrition or smoking on the child's behavioural and cognitive phenotype. However, very little is known about the specific localization of the features that, when differentially methylated, regulate gene expression and are thus relevant for epigenetic epidemiologists to study [14]. Even less is known about candidate loci than in genetic studies, hence epigenetics should avoid the hype and promises that surrounded the early days of genetic epidemiology. Against this background it makes even less sense to blindly perform candidate gene epigenetic studies in the field of child and adolescent psychiatry as long as we have no clue where in the genome we have to look.

Two other seemingly attractive fields of research are microbiomics and psycho-immunology, which we deem to be unsuccessful. There are claims that the microbiome-gutbrain axis is of substantial relevance to mood and behaviour, suggesting an important role for the gut microbiota, and since microbiota are influenced by dietary patterns, in influencing brain development, behaviour and mood 
in humans [15]. However, as long as issues of confounding by nutrition and social environment, multiple testing, and exposure definition have not been dealt with, we lack a detailed longitudinal analysis of microbiota variation and maturation. So far there is little evidence for a causal role of microbiota in the aetiology of mental health problems in children or adolescents. Likewise, the immune system is claimed to be involved in the onset of psychiatric disorders in children and adolescents. Although the first Nobel Prize for psychiatric research in 1927 was awarded to this field, large longitudinal studies of the bi-directional association with sophisticated immunological markers are lacking. Psycho-immunological findings are inconclusive, and most studies testing the associations between CRP and cytokines, which are involved in cellular and humoral immunity, are plagued by methodological problems such as confounding, selection bias and reverse causality. Perhaps the most promising way forward is Mendelian randomization studies, once GWAS of immunological markers explains sufficient variation to permit this approach.

Lastly, we think it is time to claim that the Barker hypothesis should be critically reviewed as far as the role of foetal growth as a mediator in explaining later child mental health problems is concerned $[16,17]$. The original foetal origins hypothesis postulated by David Barker states that foetal under-nutrition leads to disproportionate foetal growth and lower birth weight which programs later cardio-metabolic disease [18]. The detailed observations of foetal growth in the Generation R cohort (8) did not support the Barker hypothesis as extended to the field of mental health. The relationship between a child's birth weight and later child functioning was largely due to shared familial (genetic) confounding or due to poor control of later postnatal risk.

Epidemiology has been invaluable to the development of child and adolescent psychiatry. This special issue attests to the fact that epidemiology counts, but better is to come: the emerging new and sophisticated epidemiological technologies employed to new large-scale child cohorts, integrating neuroscience with population data, will give us exciting insights in the mechanisms underlying the aetiology of child and adolescent psychopathology.

\section{References}

1. D’Onofrio BM, Van Hulle CA, Waldman ID, Rodgers JL, Harden KP, Rathouz PJ, Lahey BB (2008) Smoking during pregnancy and offspring externalizing problems: an exploration of genetic and environmental confounds. Dev Psychopathol 20:139-164

2. Ravens-Sieberer U, Otto C, Kriston L, Rothenberger A, Dopfner M, Herpertz-Dahlmann B, Barkmann C, Schon G, Holling H,
Schulte-Markwort M, Klasen F, The BELLA study group (2015) The longitudinal BELLA study: design, methods and first results on the course of mental health problems. Eur Child Adolesc Psychiatry. doi:10.1007/s00787-014-0638-4

3. Davey Smith G (2011) Epidemiology, epigenetics and the 'Gloomy Prospect': embracing randomness in population health research and practice. Int J Epidemiol 40:537-562

4. Richter LM, Victora CG, Hallal PC, Adair LS, Bhargava SK, Fall CHD, Lee N, Martorell R, Norris SA, Sachdev HS, Stein AD, and the COHORTS Group (2011) Cohort Profile: The consortium of health oriented research in transitioning societies. Int J Epidemiol 41:621-626

5. Barrios YV, Gelaye B, Zhong Q, Nicolaidis C, Rondon MB, Garcia PJ, Sanchez PA, Sanchez SE, Williams MA (2015) Association of childhood physical and sexual abuse with intimate partner violence, poor general health and depressive symptoms among pregnant women. PLoS One. doi:10.1371/journal.pone.0116609. eCollection. 2015

6. Horta BL, Gigante DP, Gonçalves H, Dos Santos Motta J, Loret de Mola C, Oliveira IO, Barros FC, Victora CG (2015) Cohort profile update: The 1982 Pelotas (Brazil) Birth Cohort Study. Int J Epidemiol. 2015 Mar 2. pii:dyv017 (Epub ahead of print)

7. Uher R, Rutter M (2012) Basing psychiatric classification on scientific foundation: problems and prospects. Int Rev of Psychiatry 24:591-605

8. Hohmann S, Adamo N, Lahey B, Faraone SV, Banaschewski T (2015) Genetics in child and adolescent psychiatry: methodological advances and conceptual issues. Eur Child Adolesc Psychiatry. doi:10.1007/s00787-015-0702-8

9. Verhulst FC, Tiemeier H (2015) Epidemiology of child psychopathology: major milestones. Eur Child Adolesc Psychiatry. doi:10.1007/s00787-015-0681-9

10. Steinhausen HC (2015) Recent international trends in psychotropic medication prescriptions for children and adolescents. Eur Child Adolesc Psychiatry. doi:10.1007/s00787-014-0631-y

11. Timonen-Soivio L, Vanhala R, Malm H, Leivonen S, Jokiranta E, Hinkka-Yli-Salomäki S, Gissler M, Brown AS, Sourander A (2015) The association between congenital anomalies and autism spectrum disorders in a Finnish national birth cohort. Dev Med Child Neurol. doi:10.1111/dmcn.12581. Epub 2014 Sep 8

12. O'Connell ME, Boat T, Warner KE (2009). Preventing mental, emotional, and behavioral disorders among young people: progress and possibilities. National Research Council (US) and Institute of Medicine (US) Committee on the Prevention of Mental Disorders and Substance Abuse Among Children, Youth, and Young Adults: Research Advances and Promising Interventions. National Academies Press (US), Washington (DC)

13. Duncan LE (2013) Paying Attention to All Results, Positive and Negative. J Am Acad Child Adolesc Psychiatry 52:462-465

14. Heijmans BT, Hill J (2012) The seven plagues of epigenetic epidemiology. Int J Epidemiol 2012(41):74-78. doi:10.1093/ije/ dyr225

15. Reardon S (2014) Gut-brain link grabs neuroscientists. Nature 515:175-177

16. Tiesler CM, Heinrich J (2014) Prenatal nicotine exposure and child behavioural problems. Eur Child Adolesc Psychiatry 23:913-929

17. Knudsen AK, Skogen JC, Ystrom E, Sivertsen B, Tell GS, Torgersen L (2014) Maternal pre-pregnancy risk drinking and toddler behavior problems: the Norwegian Mother and Child Cohort Study. Eur Child Adolesc Psychiatry 23:901-911

18. Barker DJ (1995) Foetal origins of coronary heart disease. BMJ 311:171-174 